\title{
Korean Guideline of Glucocorticoid-induced Osteoporosis; Time to Prevent Fracture!
}

\author{
Chang-Hee Suh \\ Department of Rheumatology, Ajou University School of Medicine, Suwon, Korea
}

Glucocorticoids are commonly used to treat various chronic inflammatory diseases, including rheumatoid arthritis, systemic lupus erythematosus, inflammatory bowel disease, and chronic obstructive pulmonary disease; they are also useful as immunosuppressants in patients undergoing organ transplantation [1,2]. A study performed in 2013 reported that based on the National Health and Nutrition Examination Survey data obtained between 1999 and 2008, the prevalence of glucocorticoid use was estimated to be $1.2 \%$ in the United States [3]. Additionally, $28.8 \%$ of oral glucocorticoid users reported $>5$-year use of the medication. Although glucocorticoids are effective anti-inflammatory agents, their long-term use is associated with various detrimental effects, including those on bone, causing glucocorticoid-induced osteoporosis (GIOP). GIOP-related fractures are associated with significant morbidity and mortality. GIOP is associated with rapid bone loss. Further, although effective treatments are available to prevent fractures in these patients, GIOP remains undertreated and preventive measures are underutilized [2]. Studies performed in patients in the US have reported concomitant use of a bisphosphonate in $8.6 \%$ of oral glucocorticoids users. Moreover, $37.9 \%$ of the patients reported use of osteoporosis medications (bisphosphonates, calcitonin, calcium, hormone replacement therapies, teriparatide, or vitamin D) [3].

Long-term glucocorticoid treatment ( $>3$ months) induces rapid bone loss and increases the risk of vertebral and non-vertebral fractures [4]. Furthermore, glucocorticoid treatment predisposes patients to all types of frac- tures in a dose-dependent manner with a particularly high risk of vertebral fractures. Discontinuation of glucocorticoid therapy reduces the fracture risk, although the risk does not return to baseline levels.

Decreased bone formation serves as the critical process in the pathogenesis of GIOP. Glucocorticoids decrease the differentiation and maturation of osteoblasts, leading to a reduction in their numbers and function [5]. Additionally, excess serum glucocorticoid levels cause osteoblast apoptosis. Osteocytes also undergo apoptosis, which leads to diminished bone quality, owing to their involvement in repair of microdamage to bone. Thus, the fracture risk in patients with GIOP is higher than that in patients with postmenopausal osteoporosis even when both patient groups demonstrate the same bone mineral density (BMD). This finding could be attributed to the direct toxic effect of glucocorticoids on osteoblasts and osteocytes [6]. However, BMD is considered a useful tool for risk stratification in patients with GIOP for appropriate decision-making regarding optimal treatment and for patient follow-up over time. To determine the treatment threshold based on assessment of the risk of fracture, the fracture risk assessment tool (FRAX) was developed by the World Health Organization in 2007. This tool includes glucocorticoid therapy as an independent risk factor for the prediction of fractures.

Owing to the increased use of glucocorticoids and the consequent increase in morbidity and mortality rates secondary to long-term glucocorticoid treatment, several countries have developed guidelines for the prevention and treatment of GIOP. Recently, the American College of

Received : December 5, 2018, Accepted : December 10, 2018

Corresponding to : Chang-Hee Suh (iD http://orcid.org/0000-0001-6156-393X

Department of Rheumatology, Ajou University School of Medicine, 164 WorldCup-ro, Yeongtong-gu, Suwon 16499, Korea. E-mail : chsuh@ajou.ac.kr

Copyright (c) 2019 by The Korean College of Rheumatology. All rights reserved.

This is an Open Access article, which permits unrestricted non-commerical use, distribution, and reproduction in any medium, provided the original work is properly cited. 
Rheumatology has established guidelines in 2017 for the prevention and treatment of GIOP based on updated evidence and group consensus [7]. It is important to establish clear guidelines for the management of GIOP in Korea. The Korean Guideline for the Prevention and Treatment of Glucocorticoid-induced Osteoporosis is timely and most updated with available evidence [8].

The Korean Society for Bone and Mineral Research (KSBMR) and the Korean College of Rheumatology (KCR) have mutually developed a guideline for the treatment of GIOP by adapting previously published guidelines with a systematic review and quality assessment. This guideline recommends the assessment and monitoring of the fracture risk and the treatment of fractures during the course of glucocorticoid administration for all adults aged $\geq 19$ years. Prompt assessment of fractures should be performed preferably within 6 months of initiation of long-term glucocorticoid treatment. Fracture risk should be evaluated using FRAX with adjustments for the glucocorticoid dose, a history of osteoporotic fractures, and BMD values. Prolonged glucocorticoid use warrants annual reassessment of the fracture risk with FRAX, BMD values, and vertebral radiography. Fracture risk is classified as high, moderate, and low. The high-risk category includes patients aged $\geq 40$ years with a history of osteoporotic fractures, a BMD T-score $\leq-2.5$, FRAXbased 10-year major osteoporotic fracture risk $\geq 20 \%$, or FRAX-based 10-year hip fracture risk $\geq 3 \%$. The moderate risk category includes FRAX-based 10-year major osteoporotic fracture risk of $10 \% \sim 19 \%$ or FRAX-based 10 -year hip fracture risk $>1 \%$ and $<3 \%$. Patients with a moderate-to-high fracture risk require treatment with calcium, vitamin $\mathrm{D}$, and osteoporosis medications. The guideline recommends the use of oral bisphosphonates as first-line therapy for GIOP. Intravenous bisphosphonates, teriparatide, and denosumab are alternatives in patients who cannot be administered oral bisphosphonates. Oral bisphosphonates are preferred, and teriparatide is considered in women who demonstrate a moderate-to-high-risk of fracture and are planning to conceive. However, osteoporosis medications (except calcium and vitamin $\mathrm{D}$ ) are not recommended during pregnancy. It is recommended that discontinuation of osteoporosis medication be considered if glucocorticoid treatment is discontinued in patients with a low fracture risk.

The most significant feature of the guideline that favors its widespread use is its simplicity and feasibility in the real-world health care system in the country. Several guidelines have been published for the management of GIOP during the last decade. However, reportedly, adherence to these guidelines is low. Notably, the guideline adherence rate observed in clinical practice was only $23.3 \%$ in Japan [9]. Considering that most general physicians cannot assess fracture risk using FRAX and that osteoporosis medication is covered along with BMD and incidental fracture detection by the Korean National Health Insurance Service, it would be preferable to include a BMD T-score $\leq-1.5$ as a moderate-risk category. Because of a relative discrepancy in GIOP between BMD and the fracture risk, with a higher frequency of fractures in patients with GIOP than in patients with postmenopausal osteoporosis (even when intergroup BMD values are similar) [6], the International Osteoporosis Foundation and the European Calcified Tissue Society continue to recommend -1.5 as the cut-off BMD T-score [10]. Notably, after the publication of the Korean Guideline for the Prevention and Treatment of Glucocorticoid-induced Osteoporosis, the Health Insurance Review \& Assessment Service in Korea announced that osteoporosis medications are covered in patients who receive chronic glucocorticoids and show a BMD T-score $\leq-1.5$.

The most recent and updated Korean guideline for GIOP was developed after remarkable concerted efforts of a development committee and a working committee supported by the KSBMR and KCR. It is expected that the timely application of this guideline would effectively prevent fractures in patients receiving chronic glucocorticoid treatment.

\section{ACKNOWLEDGMENTS}

This research was supported by a grant from the Korea Health Technology R\&D Project through the Korea Health Industry Development Institute (KHIDI), funded by the Ministry of Health \& Welfare, Republic of Korea (grant number: HI16C0992).

\section{CONFLICT OF INTEREST}

No potential conflict of interest relevant to this article was reported.

\section{REFERENCES}

1. Rizzoli R, Biver E. Glucocorticoid-induced osteoporosis: who to treat with what agent? Nat Rev Rheumatol 2015; 
11:98-109.

2. Overman RA, Yeh JY, Deal CL. Prevalence of oral glucocorticoid usage in the United States: a general population perspective. Arthritis Care Res (Hoboken) 2013;65:294-8.

3. Whittier X, Saag KG. Glucocorticoid-induced osteoporosis. Rheum Dis Clin North Am 2016;42:177-89.

4. van Staa TP, Leufkens HG, Cooper C. The epidemiology of corticosteroid-induced osteoporosis: a meta-analysis. Osteoporos Int 2002;13:777-87.

5. Canalis E, Mazziotti G, Giustina A, Bilezikian JP. Glucocorticoid-induced osteoporosis: pathophysiology and therapy. Osteoporos Int 2007;18:1319-28.

6. Kaji H, Yamauchi M, Chihara K, Sugimoto T. The threshold of bone mineral density for vertebral fracture in female patients with glucocorticoid-induced osteoporosis. Endocr J 2006;53:27-34.

7. Buckley L, Guyatt G, Fink HA, Cannon M, Grossman J,
Hansen KE, et al. 2017 American College of Rheumatology guideline for the prevention and treatment of glucocorticoid-induced osteoporosis. Arthritis Care Res (Hoboken) 2017;69:1095-110.

8. Park SY, Gong HS, Kim KM, Kim D, Kim HY, Jeon CH, et al. Korean guideline for the prevention and treatment of glucocorticoid-induced osteoporosis. J Rheum Dis 2018;25:263-95.

9. Kirigaya D, Nakayama T, Ishizaki T, Ikeda S, Satoh T. Management and treatment of osteoporosis in patients receiving long-term glucocorticoid treatment: current status of adherence to clinical guidelines and related factors. Intern Med 2011;50:2793-800.

10. Lekamwasam S, Adachi JD, Agnusdei D, Bilezikian J, Boonen S, Borgström F, et al. A framework for the development of guidelines for the management of glucocorticoid-induced osteoporosis. Osteoporos Int 2012;23:2257-76. 